\title{
Strain tuning high thermal conductivity in semiconductors and polymers
}

\author{
Jivtesh Garg \\ School of Aerospace and Mechanical Engineering \\ University of Oklahoma, USA
}

This talk focuses on the role of strain in enhancing thermal conductivity in compound semiconductors and polymer/graphene nanocomposites. Phonon dispersion of compound semiconductors such as Boron Phosphide (BP) exhibits a large phonon band gap between acoustic and optical phonons. This band gap suppresses scattering of acoustic phonons by optical phonons leading to higher phonon lifetimes, thus leading to very high thermal conductivities in such compound semiconductors. In this work, we show that strain can further increase band gap, thus providing avenues to achieve even higher thermal conductivities. In particular, we show that strain can enhance thermal conductivity at nanometer length scales. Strain is shown to diminish scattering at higher frequencies, where phonon meanfreepaths are in the nanometer regime. Increase in meanfreepaths in the nanometer regime, leads to thermal conductivity enhancement at these length scales.

Strain can also enhance thermal conductivity of polymer/graphene nanocomposites by simultaneously aligning polymer lamellae and graphene nanoplatelets. In polymers, thermal conductivity is high along the chain axis, however, random orientation of these polymer chains leads to overall low thermal conductivity. Similarly, graphene nanoplatelets have high in-plane thermal conductivity approaching $\sim 1500 \mathrm{~W} / \mathrm{mK}$, but low out-of-plane thermal conductivity of the order of $\sim 10$ $\mathrm{W} / \mathrm{mK}$. By orienting both polymer chains and planar direction of graphene nanoplatelets along the direction of heat transfer, large increase in thermal conductivity can be achieved. In this work, quantitative measurements of the effect of such simultaneous alignment on thermal conductivity enhancement are reported. 